\title{
Aplicativos móveis e sua contribuição para pais sobre o desenvolvimento infantil
}

\author{
Mobile applications and their contribution for parents about child development \\ Aplicaciones móviles y su contribuición a los padres en el desarrollo infantil
}

Recebido: 27/03/2021 | Revisado: 04/04/2021 | Aceito: 12/04/2021 | Publicado: 24/04/2021

\author{
Douglas da Silva Rodrigues \\ ORCID: https://orcid.org/0000-0002-1440-2997 \\ Universidade do Estado do Pará, Brasil \\ E-mail: douglasrodrigues58@gmail.com \\ Meyson Santos Silva \\ ORCID: https://orcid.org/0000-0002-7346-6368 \\ Universidade do Estado do Pará, Brasil \\ E-mail:meeeyson@gmail.com \\ Lorena de Oliveira Tannus \\ ORCID: https://orcid.org/0000-0002-1917-0267 \\ Universidade do Estado do Pará, Brasil \\ E-mail: tannuslorena@gmail.com \\ Ivete Furtado Ribeiro Caldas \\ ORCID: https://orcid.org/0000-0002-2095-101X \\ Universidade do Estado do Pará, Brasil \\ E-mail: ivete.caldas@uepa.br
}

\begin{abstract}
Resumo
O uso de softwares para as áreas da saúde tem atingido destaque nos últimos anos. Todavia, ainda são escassos dados referentes a quantidade e as características estruturais daqueles que abordem sobre o desenvolvimento de crianças nos primeiros anos de vida e os determinantes neurobiológicos, sociais e ambientais que podem interferir nesse processo. Este estudo objetivou realizar uma investigação documental em bases de dados indexadas e em aplicativos móveis sobre orientação para pais acerca do desenvolvimento infantil. Trata-se de um estudo de investigação documental, descritivo e de abordagem quantitativa. Para a busca de conteúdo teórico-científico e de aplicativos, a pesquisa realizada ocorreu em três etapas: 1) levantamento bibliográfico utilizando os descritores em bases de dados científicas Pubmed, via Medical Literature Analysis and Retrievel System Online (MEDLINE), Cochrane Library e Biblioteca Virtual em Saúde (BVS); 2) o levantamento de aplicativos para dispositivos móveis nas lojas virtuais Google Play e App Store, nas plataformas Android e iOS, respectivamente; 3) organização dos dados. Foram encontrados 199 artigos, porém, apenas 2 artigos destacaram-se sobre a eficiência na utilização de mensagens de texto e meios telefônicos sobre orientações para gestantes e puérperas. Na plataforma App Store um total de 425 aplicativos foram encontrados, entretanto, apenas 9 atendem os critérios de inclusão e exclusão, enquanto na Google Play 1149 foram encontrados e somente 29 contemplaram os critérios. Portanto, faz-se necessário o desenvolvimento de ferramentas tecnológicas com propostas mais didáticas, interativas e com conteúdo científico para pais sobre o desenvolvimento infantil.
\end{abstract}

Palavras-chave: Desenvolvimento infantil; Nascimento prematuro; Educação em saúde; Software.

\begin{abstract}
The use of sftwares in the health areas has achieved prominence in the last few years. However, data on the quantity and structural characteristics of those who adressed the development of children in the first years of life and the neurobiological, social and environmental determimants that may interfere in this process are still scarce. This study aimed to carry out a documentary investigation in the national and international context on articles available in indexed and applications for mobile devices that can contribute as guidance for parents on child development. This is documentary, descriptive sutdy with a qualitative approach, based on the survey of indexed databases and applications for smartphones with Android and iOS operating system. For the reasearch for theoretical-scientific content and applications, an exploratory reasearch was carried out in three stages: 1) bibliographic surveys using the in scientific databases Pubmed, via Medical Literature Analysis and Retrievel System Online (MEDLINE), Cochrane Library e Virtual Health Library (VHL); 2) the survey of application for mobile devices Google Play and App Store virtual stores, in the Android and iOS platform, respectively; 3) data organization. 199 articles were found with the descriptors, however, only 2 articles stood out on the eficiency in the use of text messages and telephone means on pregnant and postpartum guidelines. On the App Store platform a total of 425 applications were found, however, only 9 met the inclusion and exclusion criteria, while on Google Play 1149 were found and only 29 met the criteria. Therefore it is necessary to develop applications for mobile devices with more didatic, interactive proposals and with scientif content about guidance for parents on child development.
\end{abstract}


Keywords: Child development; Premature birth; Health education; Software.

\section{Resumen}

El uso de software para áreas de salud há cobrado protagonismo en los últimos años. Sin embargo, aún son escasos los datos sobre la cantidad y características estructurales de quianes abordan el desarrollo de los niños em los primeros años de vida y los determinantes neurobiológicos, sociales y ambientales que pueden interferir en este proceso. Este estudio tuvo como objetivo realizar uma investigación documental en el contexto nacional y internacional sobre artículos disponibles en indexados y aplicaciones para dispositivos móviles que puedan contribuir como orientación a los padres sobre el desarrollo infantil. Se trata de um estudio documental, descriptivo con enfoque cualitativo, basado en la encuesta de bases de datos indexadas y aplicaciones para teléfonos inteligentes com sistema operativo Android e iOS. Para la búsqueda de contenidos y aplicaciones teórico-científias se realizó una investigación exploratoria en tres etapas: 1) levantamiento bibliográfico utilizando los descriptores en bases de datos científicas Pubmed, vía Medical Literature Analysis and Retrievel System Online (MEDLINE), Cochrane Library y Virtual Health Biblioteca (BVS); 2) el releviamento de aplicaciones para dispositivos móviles em ças tiendas virtuales Google Play y App Store, en las plataformas Android y iOS respectivamente; 3) organización de datos. Se encontraron 199 artículos con los descriptores, sin embargo, solo se destacaron 2 artículos sobre la eficiencia en el uso de mensajes de texto y medios telefónicos en orientaciones gestantes y puerperales. En la plataforma App Store se encontraton un total de 425 aplicaciones, sin embargo, solo 9 cumplieron com los criterios de inclusión y exclusión, mientras que en Google Play se encontraron 1149 y solo 29 cumplieron con los criterios. Por lo tanto, es necesario desarrollar aplicaciones para dispositivos móviles con propuestas más didácticas, interactivas y com contenido científico sobre orientación a los padres en el desarrollo infantil.

Palabras clave: Desarrollo infantil; Nacimiento prematuro; Educación para la salud; Software.

\section{Introdução}

O uso de softwares para as áreas tanto da educação, quanto da saúde, tem atingido destaque nos últimos anos, seja para educar ou para auxiliar em procedimentos mais complexos. Isso se deve ao acesso às tecnologias e ao crescimento dos recursos computacionais no meio acadêmico, os quais têm trazido inovações e métodos alternativos de orientações, aprendizagem e de produção de conhecimento (G. F. Souza et al., 2020; Souza Toledo \& Cardoso, 2020).

Uma das maiores vantagens no uso de softwares, trata-se da possibilidade de serem desenvolvidos como aplicativos para dispositivos móveis, como celulares, facilitando o uso, o acesso e tornando prática a mobilidade do recurso para diversos lugares, independentemente da plataforma a ser utilizada. As plataformas que possuem mais destaque atualmente, são os sistemas Android, iOS e Windows Mobile (Silva \& Santos, 2014).

Nesse contexto, o número de aplicativos desenvolvidos nas áreas da saúde vem atingindo grande importância por possuírem ferramentas didáticas, interativas e de fácil manuseio, maximizando o acesso da educação em saúde em todas as classes econômicas, e com isso, buscando minimizar as problemáticas relacionadas a saúde no dia a dia da sociedade (Tibes et al., 2014).

Todavia, ainda são escassos dados referentes a quantidade e as características estruturais de softwares que abordem sobre o desenvolvimento de crianças nos primeiros anos de vida e os determinantes neurobiológicos, sociais e ambientais que podem interferir na trajetória desse desenvolvimento, incluindo habilidades motoras, cognitivas, sociais e comunicativas (Sanchez-Joya et al., 2017). Entre esses determinantes têm-se o nascimento prematuro (Caldas et al., 2018), infecções neonatais por transmissão vertical (Zika Vírus e Sífilis) (Brogueira \& Miranda, 2017) e a qualidade da interação mãe-filho (Mesquita et al., 2020).

Nessa perspectiva, estima-se que anualmente nascem 15 milhões de crianças prematuras no mundo, e esse número vem aumentando no decorrer dos anos. A prematuridade é um forte indicador de morbimortalidade neonatal, sendo essa população vulnerável às alterações motoras e cognitivas, incluindo alterações sociocomunicativas, dificuldade de aprendizagem comparado com crianças nascidas a termo (OMS, 2021). Além do mais, um número robusto de pesquisas nacionais e internacionais apontam o efeito da qualidade na prestação de cuidados precoce dos pais na trajetória do desenvolvimento das habilidades motoras e 
comunicativas desses prematuros e crianças nos primeiros anos de vida (Caldas et al., 2018; Sanchez-Joya et al., 2017; Shah et al., 2013).

Dessa forma, o conhecimento adquirido com o uso das ferramentas tecnológicas pode reduzir os impactos negativos do desenvolvimento na vida da criança, além de proporcionar efeito positivo na qualidade de vida de seus pais (Cunha et al., 2021), permitindo o acesso às orientações quanto ao desenvolvimento esperado para os primeiros anos de vida e à compreensão de conceitos e identificação precoce de déficits e/ou atrasos no desenvolvimento da criança. Portanto, este estudo objetivou realizar uma investigação documental em bases de dados indexadas e em aplicativos móveis sobre orientação para pais acerca do desenvolvimento infantil.

\section{Metodologia}

Trata-se de um estudo documental, descritivo e de abordagem quantitativa, a partir do levantamento de bases de dados científicas e de aplicativos para smartphones com o sistema operacional Android e iOS. Esse estudo faz parte do projeto intitulado

“Aplicativo móvel para pais e/ou cuidadores sobre o desenvolvimento infantil” aprovado no Comitê de Ética em Pesquisa (Parecer: 4.218.175) que objetivou desenvolver e validar um aplicativo para pais e/ou cuidadores contendo orientações sobre desenvolvimento infantil.

Estudos potenciais foram identificados por meio de uma estratégia abrangente, da seguinte forma: (1) ("Child Development") OR (Development, Child) OR (Infant Development) OR (Development, Infant) AND (2) ("Premature Birth") OR (Birth, Premature) OR (Births, Premature) OR (Premature Births) OR (Preterm Birth) OR (Birth, Preterm) OR (Births, Preterm) OR (Preterm Births) AND (3) ("Software") OR (Computer Software) OR (Software, Computer) OR (Computer Programs) OR (Computer Program) OR (Program, Computer) OR (Programs, Computer) OR (Software Tools) OR (Software Tool) OR (Tool, Software) OR (Tools, Software) OR (Software Engineering) OR (Engineering, Software) OR (Computer Applications Software) OR (Applications Software, Computer) OR (Applications Softwares, Computer) OR (Computer Applications Softwares) OR (Software, Computer Applications) OR (Softwares, Computer Applications) OR (Computer Software Applications) OR (Application, Computer Software) OR (Applications, Computer Software) OR (Computer Software Application) OR (Software Application, Computer) OR (Software Applications, Computer) OR (Computer Programs and Programming) AND (4) ("Health Education") OR (Education, Health) OR (Community Health Education) OR (Education, Community Health) OR (Health Education, Community)

Para a busca de conteúdo teórico-científico e de aplicativos, a pesquisa realizada ocorreu em três etapas descritas a seguir. 1 Etapa:

Realizada a revisão bibliográfica utilizando os seguintes descritores: (Child development) e (Premature birth) e(Software) e (Health education). Para o refinamento adequado da pesquisa foram definidos como critérios de inclusão, artigos indexados nas bases de dados Pubmed, via Medical Literature Analysis and Retrievel System Online (MEDLINE), Cochrane Library e Biblioteca Virtual em Saúde (BVS) disponíveis em formato eletrônico, na íntegra, em português e inglês, no período de 2010 a 2020. Foram excluídos relatos de caso e duplicados nas bases consultadas. A estratégia de busca envolveu o cruzamento de palavras-chave selecionadas com base nos Medical Subject Headings (Mesh) e no Descritores de Ciências da Saúde (Decs).

Em seguida, os artigos foram organizados para ponderar o rigor e as características de cada um deles, observando suas convergências e divergências na comparação com os demais achados. Para a apresentação das etapas de seleção dos artigos utilizou-se o modelo Preferred Reporting Items for Systematic Reviews and Meta-Analyses (PRISMA) (Moher et al., 2009) de acordo com a Figura 1: 
Figura 1. Fluxograma Prisma.

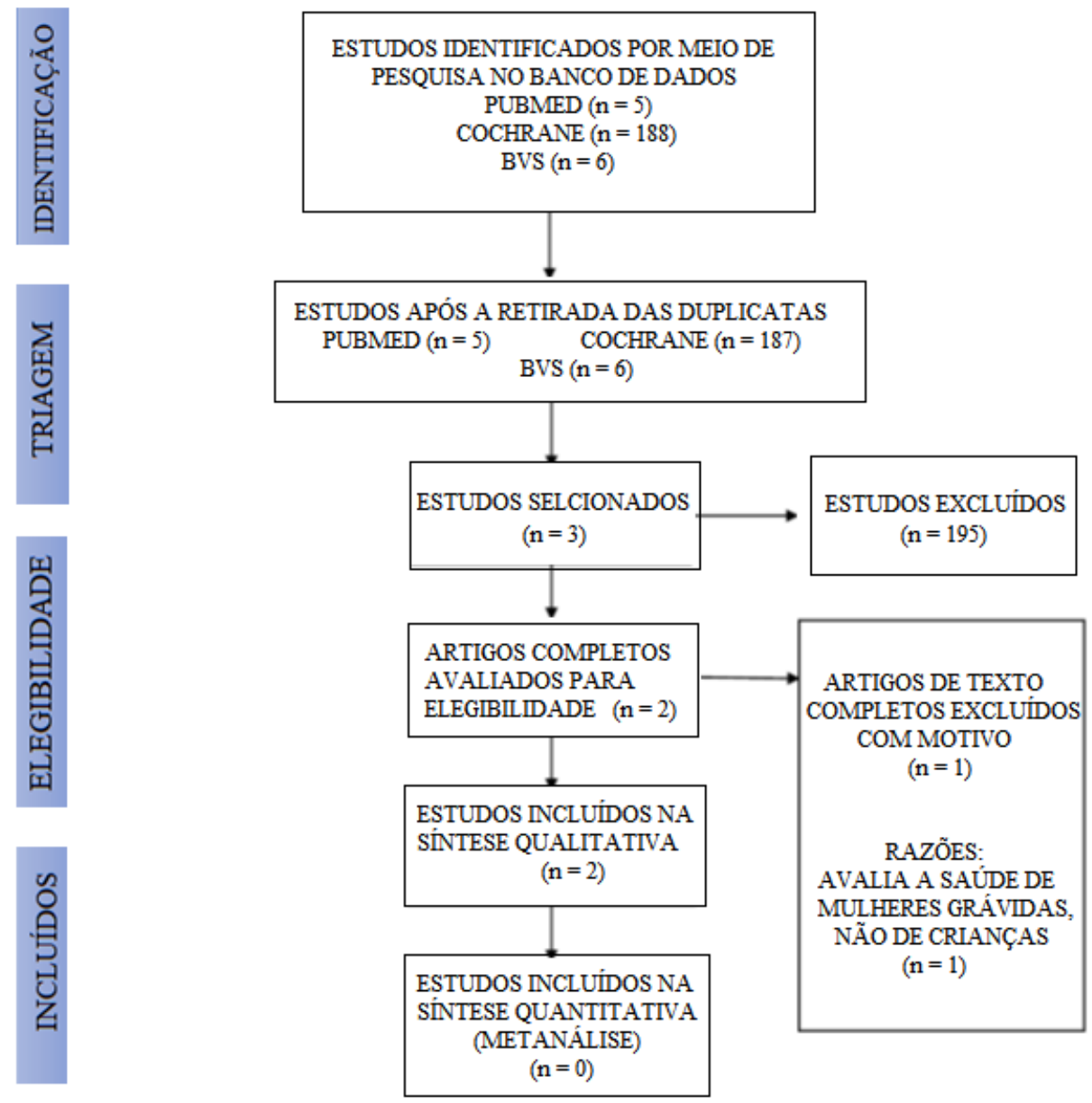

Fonte: Moher et al (adaptado) (2009).

Para a apreciação e síntese dos artigos selecionados para este estudo, foi construído um quadro sinóptico contemplando os seguintes aspectos: título do artigo; autor (es); ano da publicação; periódico; instrumento; desfecho de acordo com o Quadro 1:

Quadro 1. Dados dos artigos.

\begin{tabular}{|c|c|c|c|c|}
\hline \multicolumn{5}{|c|}{ Dados dos Artigos } \\
\hline Título & Autor & Ano & Instrumento & Desfecho \\
\hline $\begin{array}{l}\text { Mobile phone } \\
\text { messaging for } \\
\text { preventive health } \\
\text { care }\end{array}$ & $\begin{array}{l}\text { Vodopivec-Jamsek, } \\
\text { Vlasta } \\
\text { de Jongh, Thyra } \\
\text { Gurol-Urganci, Ipek } \\
\text { Atun, Rifat } \\
\text { Car, Josip }\end{array}$ & 2012 & $\begin{array}{l}\text { Intervenções usando } \\
\text { SMS e MMS }\end{array}$ & $\begin{array}{c}\text { Devido ao pequeno número de } \\
\text { participantes em três dos estudos } \\
\text { incluídos, combinado com as limitações do } \\
\text { estudo de risco de viés e falta de } \\
\text { causalidade demonstrada, a evidência para } \\
\text { esses efeitos é de baixa a moderada } \\
\text { qualidade. }\end{array}$ \\
\hline $\begin{array}{l}\text { Telephone } \\
\text { support for } \\
\text { women during } \\
\text { pregnancy and the } \\
\text { first six weeks } \\
\text { postpartum }\end{array}$ & $\begin{array}{l}\text { Lavender, Tina } \\
\text { Richens, Yana } \\
\text { Milan, Stephen J. } \\
\text { Smyth, Rebecca M.D. } \\
\text { Dowswell, Therese }\end{array}$ & 2013 & $\begin{array}{l}\text { Pesquisa em bases de } \\
\text { dados }\end{array}$ & $\begin{array}{c}\text { Há evidências insuficientes para } \\
\text { recomendar suporte telefônico de rotina } \\
\text { para mulheres com acesso à maternidade } \\
\text { serviços. }\end{array}$ \\
\hline
\end{tabular}


2a Etapa:

Fase em que ocorreu a busca de aplicativos móveis disponíveis nas lojas virtuais Google Play e Play Store. Para a busca foram utilizadas as palavras chave "Development", Developmental medicine", "Child development", "Parenthing", "Health e parenthing", onde constatou-se aplicativos disponíveis sobre o tema, para os sistemas operacionais Android e IOS.

Quanto aos critérios de inclusão dos aplicativos foram selecionados Apps gratuitos, disponíveis em português e inglês e com classificação acima de três estrelas pelos usuários. Sendo excluídos aqueles aplicativos que aparecem repetidos em mais de um descritor, aplicativos pagos, em outros idiomas e que não se enquadram nos objetivos da pesquisa. $3^{\text {a }}$ Etapa:

Foi realizada a organização dos dados e posteriormente transcritos em forma de textos e imagens.

\section{Resultados e Discussão}

Dentre os periódicos que publicaram sobre o uso de ferramentas tecnológicas, destacou-se a Cochrane Database of Systematic Reviews com 2 artigos, cuja ideia central captada de cada artigo foi registrada de acordo com o Quadro 2:

Quadro 2. Ideia central dos artigos.

\begin{tabular}{|l|c|c|}
\hline \multicolumn{1}{|c|}{ Ideia Central dos Artigos } \\
\hline \multicolumn{1}{|c|}{ Título } & Autor & Objetivo \\
\hline $\begin{array}{l}\text { Mobile phone messaging for } \\
\text { preventive health care }\end{array}$ & $\begin{array}{c}\text { Vodopivec-Jamsek, Vlasta } \\
\text { de Jongh, Thyra } \\
\text { Gurol-Urganci, Ipek } \\
\text { Atun, Rifat } \\
\text { Car, Josip }\end{array}$ & $\begin{array}{c}\text { Avaliar os efeitos das intervenções de mensagens de } \\
\text { telefone móvel como um modo de entrega para } \\
\text { cuidados de saúde preventivos, no estado de saúde e no } \\
\text { desfecho em comportamento de saúde }\end{array}$ \\
\hline $\begin{array}{l}\text { Telephone support for } \\
\text { women during pregnancy } \\
\text { and the first six weeks } \\
\text { postpartum }\end{array}$ & Lavender, Tina \\
Richens, Yana & Milan, Stephen J. \\
Smyth, Rebecca M.D. & Dowswell, Therese & $\begin{array}{c}\text { Avaliar os efeitos do suporte telefônico durante a } \\
\text { gravidez e nas primeiras seis semanas pós-parto, em } \\
\text { comparação com o atendimento de rotina, nos } \\
\text { resultados maternos e infantis }\end{array}$ \\
\hline
\end{tabular}

Fonte: Autores.

No estudo de Vodopivec-Jamsek et al, pode-se verificar como o uso das ferramentas tecnológicas móveis em saúde preventiva pode ser positiva, destacando-se a utilização de mensagens de texto para auxiliar gestantes durante o pré-natal. A maioria das participantes alegou níveis satisfatórios no uso da ferramenta pela agilidade em relação às orientações recebidas via mensagem de texto, contribuindo de forma positiva para saúde física e mental, além de diminuir os níveis de ansiedade e estresse nesse período. O estudo ressalta também a importância dessas orientações para o desenvolvimento de bons hábitos de vida, como cessação do tabagismo. Da mesma forma, no estudo de Lavender et al, foram utilizados meios telefônicos para o contato com as puérperas, com o propósito de orientá-las sobre a amamentação e prevenir casos de depressão pós-parto, sendo esse tipo de intervenção considerado um modo promissor.

Em relação aos aplicativos móveis, foram encontrados na plataforma Play Store um total de quatrocentos e vinte e cinco (425) aplicativos, duzentos e nove (209) com o descritor "development", vinte e cinco (25) com "developmental medicine", cento e noventa (190) com "child development", um (1) com "parenthing" e nenhum com "health parenthing". Entretanto, apenas nove (9) atenderam aos critérios de inclusão e exclusão. Na plataforma Google Play foram encontrados mil cento e quarenta e nove (1149) aplicativos, duzentos e cinquenta (250) pelo descritor "development", duzentos e quarenta e nove (249) pelo 
“Developmental Medicine", duzentos e cinquenta (250) pelo "Health Parething”, cento e cinquenta (150) pelo "Parenthing" e duzentos e cinquenta (250) pelo "Child Development". Todavia, somente vinte e nove (29) aplicativos contemplaram os critérios de inclusão e exclusão. Vale ressaltar que, de todos os aplicativos encontrados, alguns se repetem nas plataformas, totalizando um quantitativo de trinta e três (33) aplicativos selecionados. Mais da metade dos aplicativos excluídos eram pagos e/ou estavam com classificação abaixo de três estrelas pela avaliação dos usuários, sendo que alguns estavam com classificação sem nenhuma estrela por não terem recebido avaliação em quantitativo suficiente para receber esses itens avaliativos. As informações foram organizadas no Quadro 3 e na Figura 2 abaixo:

Quadro 3. Informações sobre os aplicativos para dispositivos móveis

\begin{tabular}{|c|c|c|}
\hline Nome do Aplicativo & Proposta & $\begin{array}{l}\text { Platafor } \\
\text { ma }\end{array}$ \\
\hline Baby Care week by week.Tips & $\begin{array}{l}\text { Oferece informações sobre cuidados e desenvolvimento infantil durante o primeiro } \\
\text { ano de vida. }\end{array}$ & Android \\
\hline $\begin{array}{l}\text { Growth Chart, Development } \\
\text { Milestones \& Vaccination }\end{array}$ & $\begin{array}{l}\text { Aplicativo para pais e aplicativo para rastreador de bebês: Seções simplificadas } \\
\text { para gráfico de crescimento, marcos de desenvolvimento, rastreador de alimentos, } \\
\text { vacinação e dica de saúde. }\end{array}$ & Android \\
\hline $\begin{array}{l}\text { Baby Tips: The Ultimate } \\
\text { Parental Guide }\end{array}$ & $\begin{array}{l}\text { Dicas algumas vezes por semana sobre o desenvolvimento do bebê, saúde, } \\
\text { nutrição, esportes, educação e psicologia do bebê }\end{array}$ & Android \\
\hline $\begin{array}{l}\text { Babygogo Parenting - Baby } \\
\text { Care \& Pregnancy Tips }\end{array}$ & $\begin{array}{l}\text { Aplicativo que garante comunicação com profissionais e informações sobre } \\
\text { crescimento e desenvolvimento. }\end{array}$ & Android \\
\hline Child Development 7-12 years & $\begin{array}{l}\text { Aplicativo para pais ou profissionais a respeito do desenvolvimento das crianças } \\
\text { dos } 7 \text { a } 12 \text { anos. }\end{array}$ & Android \\
\hline Child Development & $\begin{array}{l}\text { Permite a pais e educadores acompanhar o desenvolvimento infantil para crianças } \\
\text { desde o nascimento até a idade de } 5 \text { anos. }\end{array}$ & Android \\
\hline $\begin{array}{l}\text { Parenting and Child Learning } \\
\text { Development }\end{array}$ & $\begin{array}{l}\text { O aplicativo fornece ideias, dicas, estratégias de disciplina e habilidades práticas } \\
\text { para auxiliar no desenvolvimento da criança, ajudando pais no processo de } \\
\text { desenvolvimento. }\end{array}$ & Android \\
\hline Child Learning Development & $\begin{array}{l}\text { Fornece ideias, dicas e habilidades práticas para auxiliar no desenvolvimento das } \\
\text { crianças por meio da educação infantil em todas as áreas de sua vida. }\end{array}$ & Android \\
\hline $\begin{array}{l}\text { Child Development Stages } \\
\text { Guide }\end{array}$ & Dicas e informações sobre os estágios do desenvolvimento & Android \\
\hline CDC Milestone Tracker & $\begin{array}{l}\text { Permite acompanhar os marcos de crianças nos principais estágios de } \\
\text { desenvolvimento de } 2 \text { meses a } 5 \text { anos com as listas de verificação ilustradas do } \\
\text { CDC }\end{array}$ & Android \\
\hline Baby Growth \& Development & $\begin{array}{l}\text { Auxilia pais sobres marcos do desenvolvimento e parâmetros de acordo com a } \\
\text { idade. }\end{array}$ & Android \\
\hline $\begin{array}{l}\text { Baby Tracker - Newborn } \\
\text { Feeding, Sleep, Diaper }\end{array}$ & Oferece informações semanais e mensais sobre o desenvolvimento da criança. & Android \\
\hline $\begin{array}{c}\text { Child Development Stages } \\
\text { Guide }\end{array}$ & Guia de marcos do desenvolvimento infantil. & Android \\
\hline $\begin{array}{l}\text { Child Development } \\
\text { Milestones }\end{array}$ & $\begin{array}{l}\text { Fornece uma lista abrangente de marcos de desenvolvimento infantil, incluindo } \\
\text { desenvolvimento físico normal, atividades que promovem o crescimento saudável } \\
\text { e preocupações com o desenvolvimento. }\end{array}$ & Android \\
\hline Baby Development Guide & $\begin{array}{l}\text { Guia de desenvolvimento para pais e familiares acompanharem os marcos no } \\
\text { desenvolvimento de um bebê, desde o nascimento até os primeiros anos da } \\
\text { infância. }\end{array}$ & Android \\
\hline
\end{tabular}




\begin{tabular}{|c|c|c|}
\hline $\begin{array}{l}\text { Continua Kids - Child } \\
\text { Growth \& Development }\end{array}$ & $\begin{array}{l}\text { Aplicativo que fornece os marcos do desenvolvimento do bebê e os sinais de } \\
\text { atrasos no desenvolvimento e informações sobre terapias de intervenção. }\end{array}$ & Android \\
\hline $\begin{array}{l}\text { Child Development } \\
\text { Milestones }\end{array}$ & $\begin{array}{l}\text { Fornece uma lista abrangente de marcos de desenvolvimento infantil, incluindo } \\
\text { desenvolvimento físico normal, atividades que promovem o crescimento saudável } \\
\text { e questões de desenvolvimento. }\end{array}$ & Android \\
\hline $\begin{array}{l}\text { Baby Development Week by } \\
\text { Week }\end{array}$ & Diário com conteúdo informativo sobre desenvolvimento. & Android \\
\hline $\begin{array}{l}\text { The Happy Child - Parenting } \\
\text { App }\end{array}$ & $\begin{array}{l}\text { Oferece acesso a pesquisas de ponta e dicas sobre o desenvolvimento infantil e } \\
\text { como estimulá-lo. }\end{array}$ & Android \\
\hline $\begin{array}{l}\text { Child Development \& } \\
\text { Parenting News | Community }\end{array}$ & $\begin{array}{l}\text { Aplicativo fornece pesquisas mais recentes em desenvolvimento infantil para pais } \\
\text { e cuidadores. }\end{array}$ & Android \\
\hline $\begin{array}{l}\text { mSpark - Early Childhood } \\
\text { Care and Development }\end{array}$ & $\begin{array}{l}\text { Plataforma abrangente para o desenvolvimento holístico e as necessidades de } \\
\text { intervenção de crianças na primeira infância. }\end{array}$ & Android \\
\hline Bee Parenting & $\begin{array}{l}\text { Oferece dicas e soluções para os pais para os desafios diários dos pais, com } \\
\text { curadoria de especialistas na área de Desenvolvimento na Primeira Infância. }\end{array}$ & Android \\
\hline WebMD Baby & $\begin{array}{l}\text { Aplicativo para acompanhar o desenvolvimento de seus bebês e obter conselhos } \\
\text { confiáveis e aprovados por médicos }\end{array}$ & Android \\
\hline $\begin{array}{l}\text { Kinedu: desenvolvimento do } \\
\text { seu bebê }\end{array}$ & $\begin{array}{l}\text { Oferece planos de atividades personalizadas, informação sobre os marcos do } \\
\text { desenvolvimento, artigos relacionados a saúde e atividades divertidas, além de } \\
\text { dicas para criar uma base solidas para o desenvolvimento do recém-nascido. }\end{array}$ & $\begin{array}{l}\text { Android e } \\
\quad \text { iOS }\end{array}$ \\
\hline Bebe + & Oferece registro de atividades de desenvolvimento e informações para cuidadores. & $\begin{array}{l}\text { Android e } \\
\text { iOS }\end{array}$ \\
\hline $\begin{array}{c}\text { Etapas do desenvolvimento } \\
\text { infantil }\end{array}$ & Traz informações para cuidadores a respeito do desenvolvimento infantil. & Android \\
\hline $\begin{array}{c}\text { BabyCentre - Pregnacy } \\
\text { trackerMeu Bebê }\end{array}$ & $\begin{array}{l}\text { Oferece um guia para gestantes sobre as etapas da gestação e, quando a criança } \\
\text { nasce, o app se torna um guia com dicas sobre o primeiro ano de vida. Dispõe do } \\
\text { salvamento de registros, informações e dicas sobre desenvolvimento. }\end{array}$ & $\begin{array}{c}\text { iOS } \\
\text { Android }\end{array}$ \\
\hline $\begin{array}{l}\text { BabySparks - developmente } \\
\text { appBabyCentre - Pregnacy } \\
\text { tracker }\end{array}$ & $\begin{array}{l}\text { Age como um suporte para o desenvolvimento da criança através de dicas de } \\
\text { estímulos e vídeos e imagens interativos. Oferece um guia para gestantes sobre as } \\
\text { etapas da gestação e, quando a criança nasce, o app se torna um guia com dicas } \\
\text { sobre o primeiro ano de vida. }\end{array}$ & $\mathrm{iOS}$ \\
\hline $\begin{array}{l}\text { Baby exercises and } \\
\text { activitiesBabySparks - } \\
\text { developmente app }\end{array}$ & $\begin{array}{l}\text { Estimula o desenvolvimento da criança através de exercícios em vídeos. Age como } \\
\text { um surporte para o desenvolvimento da criança através de dicas de estímulos e } \\
\text { vídeos e imagens interativos }\end{array}$ & $\begin{array}{l}\text { iOS e } \\
\text { Android }\end{array}$ \\
\hline $\begin{array}{l}\text { IK: Super Parents, } \\
\text { ParentingBaby exercises and } \\
\text { activities }\end{array}$ & $\begin{array}{l}\text { Aplicativo voltado para os pais, os quais poderão aprender a lidar com ciúmes e } \\
\text { estresse na criança.Estimula o desenvolvimento da criança através de exercícios } \\
\text { em vídeos }\end{array}$ & iOS \\
\hline $\begin{array}{l}\text { Playfully baby development } \\
\text { appIK: Super Parents, } \\
\text { Parenting }\end{array}$ & $\begin{array}{l}\text { Aplicativo com ideias de exercícios para estimular a criança de } 0 \text { a } 3 \text { anos de } \\
\text { idadeAplicativo voltado para os pais, os quais poderão aprender a lidar com ciúmes } \\
\text { e estresse na criança. }\end{array}$ & $\begin{array}{l}\text { iOS e } \\
\text { Android }\end{array}$ \\
\hline $\begin{array}{l}\text { Lyfeline Milestone: baby } \\
\text { developmentPlayfully baby } \\
\text { development app }\end{array}$ & $\begin{array}{l}\text { Ajuda a detectar atrasos no desenvolvimento infantil com atividades } \\
\text { desenvolvidas por especialistas. Aplicativo com ideias de exercícios para estimular } \\
\text { a criança de } 0 \text { a } 3 \text { anos de idade }\end{array}$ & $\mathrm{iOS}$ \\
\hline $\begin{array}{l}\text { Lyfeline Milestone: baby } \\
\text { development }\end{array}$ & $\begin{array}{l}\text { Ajuda a detectar atrasos no desenvolvimento infantil com atividades } \\
\text { desenvolvidas por especialistas }\end{array}$ & iOS \\
\hline
\end{tabular}


Figura 2. Fluxograma com os artigos encontrados e selecionados.

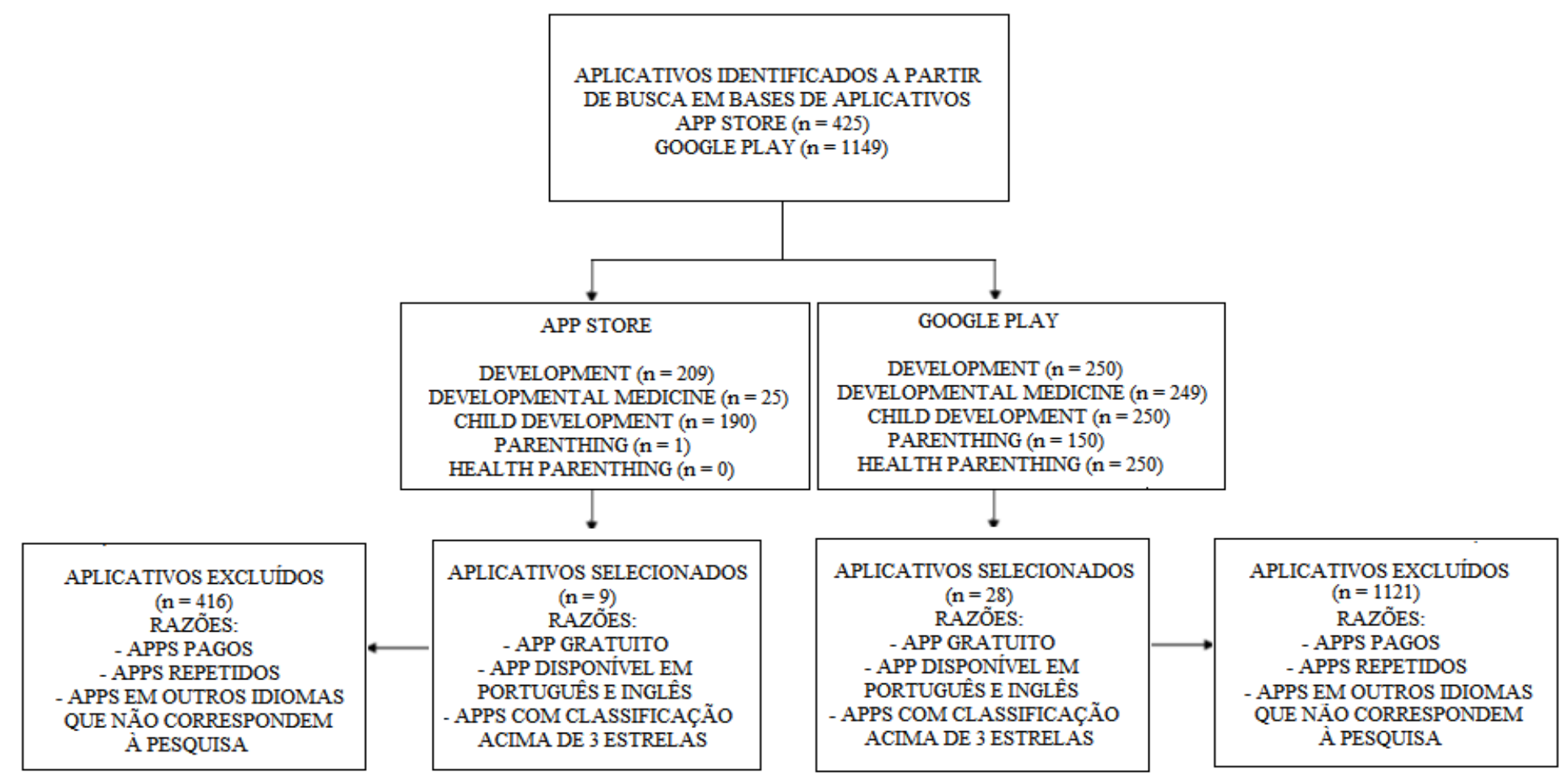

Fonte: Autores. 
Tais resultados mostram que apesar da tecnologia proporcionar o acesso à informações rápidas e práticas em diferentes dispositivos (Barra et al., 2017; Fonseca de Oliveira \& De Menezes Alencar, 2017), ainda há pouca diversidade de aplicativos específicos para orientações de pais acerca do desenvolvimento de seus filhos nos primeiros anos de vida (Armstrong et al., 2017). Geralmente os aplicativos possuem informações sobre o desenvolvimento humano e sobre os principais marcos do desenvolvimento infantil, apenas como forma de complemento às funcionalidades do aplicativo.

As demais, são limitadas as informações com rigor científico e atualizado sobre o desenvolvimento infantil e os fatores determinantes que podem interferir nesse processo, como prematuridade, infecção neonatal e qualidade na interação mãe-filho (Pereira, Flávia Monteiro; Landeira-Fernandez, 2020). Verificou-se, também, uma quantidade expressiva de aplicativos na língua inglesa comparado a portuguesa, podendo esse ser um fator determinante em questões de acessibilidade para pais brasileiros, em especial os que possuem baixas condições socioeconômicas, já que a desigualdade social ainda é uma problemática muito presente no país (Carvalho \& Souza, 2021) e visto que a formação complementar na língua inglesa é restrita, podendo limitar o acesso à informação nos meios digitais.

Por fim, outro ponto importante diz respeito a usabilidade e design do aplicativo, verificando-se uma variabilidade considerável entre eles, alguns apresentam conteúdos apenas com textos informativos e limitados recursos audiovisuais, enquanto outros, com configurações complexas e sofisticadas, porém com limitado acesso imediato, reduzindo a qualidade da interface e prejudicando a experiência dos usuários (Rocha \& Baranauskas, 2003). Nesse sentido, embora existam alguns aplicativos sobre o desenvolvimento infantil, a maioria apresenta lacunas tanto na projeção quanto no conteúdo informativo, o pode limitar a eficiência da aprendizagem acerca da finalidade ao qual o aplicativo se propõe e para o público ao qual é destinado (Bezerra et al., 2019; Rezende et al., 2016).

\section{Conclusão}

Diante do exposto, observou-se que, o uso de tecnologias na área da saúde representa um grande avanço tecnológico, o qual permite auxiliar e orientar pais acerca do cuidado e da prevenção de diversas problemáticas socioeconômicas, como aquelas advindas dos determinantes que afetam o desenvolvimento infantil. No entanto, os recursos disponíveis em formato de aplicativos móveis ainda possuem limitações importantes, como idioma restrito, valor para o acesso e conteúdo com embasamento científico.

Desse modo, é necessário investir em novas propostas de aplicativos que reúnam informações com rigor científico atualizado, com no mínimo dois idiomas, preços acessíveis, podendo, inclusive, ser disponibilizado gratuitamente e que considere diferentes determinantes que possam influenciar o processo do desenvolvimento infantil, principalmente, no tocante às características regionais e culturais.

O desenvolvimento na infância ocorre por meio de estímulos que geram diferentes sensações, portanto, utilizar ferramentas tecnológicas adequadas com a finalidade de minimizar e/ou evitar fatores que influenciam na aquisição de novas habilidades nos primeiros anos de vida são estratégias promissoras.

\section{Referências}

Armstrong, K. A., Coyte, P. C., Brown, M., Beber, B., \& Semple, J. L. (2017). Effect of home monitoring via mobile app on the number of in-person visits following ambulatory surgery a randomized clinical trial. JAMA Surgery, 152(7), 622-627. https://doi.org/10.1001/jamasurg.2017.0111

Barra, D. C. C., Paim, S. M. S., dal Sasso, G. T. M., \& Colla, G. W. (2017). Methods for developing mobile apps in health: An integrative review of the literature. Texto e Contexto Enfermagem, 26(4), 1-12. https://doi.org/10.1590/0104-07072017002260017

Bezerra, F., Silva, H., Oliveira, C., \& Brasileiro, I. (2019). Desenvolvimento de tecnologia educativa sobre desempenho neuromotor de crianças. Revista de Saúde Digital e Tecnologias Educacionais, 4(2), 46-57. https://doi.org/10.36517/resdite.v4.n2.2019.a4

Brogueira, P., \& Miranda, A. C. (2017). Vírus Zika : Emergência de um Velho Conhecido Zika Virus : Emergence of an Old Known Resumo Abstract. Revista de Sociedade Portuguesa de Medicna Interna, 146-153. http://www.spmi.pt/revista/vol24/vol24_n2_2017_146_153.pdf 
Carvalho, A. R. De., \& Souza, L. R. De. (2021). A evolução conceitual da desigualdade e da pobreza no pensamento econômico. Brazilian Journal of Political Economy, 41(2), 402-425. https://doi.org/10.1590/0101-31572021-3142

Caldas, I. F. R., Garotti, M. F., Shiramizu, V. K. M., \& Pereira, A. (2018). The socio-communicative development of preterm infants is resistant to the negative effects of parity on maternal responsiveness. Frontiers in Psychology, 9(FEB), 1-8. https://doi.org/10.3389/fpsyg.2018.00043

Cunha, K. D. C., Caldas, I. F. R., Pontes, F. A. R., Ramos, E. M. L. S., Souza, P. B. M. D., \& Silva, S. S. D. C. (2021). Qualidade da Coparentalidade e o Estresse em Pais de Crianças com Paralisia Cerebral. Revista Brasileira de Educação Especial, 27

Fonseca de Oliveira, A. R., \& De Menezes Alencar, M. S. (2017). O uso de aplicativos de saúde para dispositivos móveis como fontes de informação e educação em saúde. RDBCI: Revista Digital de Biblioteconomia e Ciência da Informação, 15(1), 234. https://doi.org/10.20396/rdbci.v0i0.8648137

Lavender, T., Richens, Y., Milan, S. J., Smyth, R. M. D., \& Dowswell, T. (2013). Telephone support for women during pregnancy and the first six weeks postpartum. Cochrane Database of Systematic Reviews, 2013(7). https://doi.org/10.1002/14651858.CD009338.pub2

Mesquita, P. C., Siqueira, D., Garotti, M., \& Caldas, I. F. (2020). Associations between maternal responsiveness due to the number of offspring and motor development. Psicologia: teoria e prática, 22(1), 144-160. https://doi.org/10.5935/1980-6906/psicologia.v22n1p144-160

Moher, D., Liberati, A., Tetzlaff, J., Altman, D. G., Altman, D., Antes, G., Atkins, D., Barbour, V., Barrowman, N., Berlin, J. A., Clark, J., Clarke, M., Cook, D., D’Amico, R., Deeks, J. J., Devereaux, P. J., Dickersin, K., Egger, M., Ernst, E., \& Tugwell, P. (2009). Preferred reporting items for systematic reviews and meta-analyses: The PRISMA statement. PLoS Medicine, 6(7). https://doi.org/10.1371/journal.pmed.1000097

Organização Mundial da Saúde [homepage na internet]. Organização Mundial da Saúde. https://www.paho.org/bra/

Pereira, F. M., \& Landeira-Fernandez, J. (2020). Construção e evidência de validade de conteúdo para aplicativo de acompanhamento e rastreio para atraso do desenvolvimento infantil. Dissertação de Mestrado - Departamento de Psicologia, Pontifícia Universidade Católica do Rio de Janeiro, 92.

Rezende, L. C. M., dos Santos, S. R., \& Medeiros, A. L. (2016). Avaliação de um protótipo para sistematização da assistência de enfermagem em dispositivo móvel. Revista Latino-Americana de Enfermagem, 24. https://doi.org/10.1590/1518-8345.0898.2714

Rocha, H. V. Da., \& Baranauskas, M. C. C. (2003). Design e avaliação de interfaces humano-computador. Interfaces, 257. http://200.18.252.196:8080/pergamumweb/vinculos/000000/000000ce.pdf

Sanchez-Joya, M. del M., Sanchez-Labraca, N., Roldan-Tapia, M. D., Moral Rodríguez, T., Ramos Lizana, J., \& Roman, P. (2017). Neuropsychological assessment and perinatal risk: A study amongst very premature born 4- and 5-year old children. Research in Developmental Disabilities, 69(March), 116-123. https://doi.org/10.1016/j.ridd.2017.08.008

Shah, P. E., Robbins, N., Coelho, R. B., \& Poehlmann, J. (2013). The paradox of prematurity: The behavioral vulnerability of late preterm infants and the cognitive susceptibility of very preterm infants at 36 months post-term. Infant Behavior and Development, 36(1), 50-62. https://doi.org/10.1016/j.infbeh.2012.11.003

Silva, M. M. da, \& Santos, M. T. P. (2014). Os Paradigmas de Desenvolvimento de Aplicativos para Aparelhos Celulares. T.I.S - Tecnologias, Infraestrutura e Software - UFSCar, 3(2), 162-170. http://revistatis.dc.ufscar.br/index.php/revista/article/view/86/80

Souza, G. F., Oliveira, É., Crosewski, N., Bonete, G., \& Dias, F. (2020). Criação de um aplicativo de estudo para residência médica baseado na perspectiva do usuário. Revista de Saúde Digital e Tecnologias Educacionais, 5(2), 30-43. https://doi.org/10.36517/resdite.v5.n2.2020.a3

Souza Toledo, M. V., \& Cardoso, A. M. P. (2020). O Uso de Softwares na Educação. Brazilian Journal of Development, 6(1), 2055-2066. https://doi.org/10.34117/bjdv6n1-147

Tibes, C. M. dos S., Dias, J. D., \& Zem-Mascarenhas, S. H. (2014). Mobile applications developed for the health sector in Brazil: an integrative literature review. REME: Revista Mineira de Enfermagem, 18(2), 471-478. https://doi.org/10.5935/1415-2762.20140035

Vodopivec-Jamsek, V., de Jongh, T., Gurol-Urganci, I., Atun, R., \& Car, J. (2012). Mobile phone messaging for preventive health care. Cochrane Database of Systematic Reviews, 2017(12). https://doi.org/c 